\title{
Apolipoprotein B and non-high-density lipoprotein cholesterol reveal a high atherogenicity in individuals with type 2 diabetes and controlled low-density lipoprotein-cholesterol
}

\author{
Liliana Fonseca ${ }^{1 *} \mathbb{D}$, Sílvia Paredes ${ }^{2}$, Helena Ramos ${ }^{1}$, José Carlos Oliveira ${ }^{3}$ and Isabel Palma ${ }^{1}$
}

\begin{abstract}
Background: Lipid-lowering therapy is guided by Low-density-lipoprotein cholesterol (LDL-c) levels, although the cardiovascular disease (CVD) risk could be better reflected by other lipid parameters. This study aimed at comparing a comprehensive lipid profile between patients with type 2 diabetes mellitus (T2DM) with LDL-c concentration within and above target.

Methods: A comprehensive lipid profile was characterized in 96 T2DM patients. The European Society of Cardiology/European Atherosclerosis Society (ESC/EAS) 2016 and 2019 Guidelines for the Management of Dyslipidemias were used to define LDL-c targets.

Results: In this population, only 28.1 and $16.7 \%$ of patients had mean LDL-c levels within target, as defined by the 2016 and 2019 guidelines, respectively. Applying the 2016 guidelines criteria, in patients with LDL-c within target, 22, 25 and 44\% presented non-high-density lipoprotein cholesterol (non-HDL-c), Apolipoprotein B (ApoB) and oxidized LDL-c levels above the recommended range, respectively, whereas according to the 2019 guidelines criteria, 50, 39 and 44\% of the patients with LDL-c within target had elevated high-density lipoprotein cholesterol $(\mathrm{HDL}-\mathrm{c}), \mathrm{ApoB}$ and oxidized LDL-c levels, respectively. LDL-c was strongly correlated with non-HDL-c $(r=0.850)$, ApoB $(r=0.656)$ and oxidized LDL-c $(r=0.508)$. Similarly, there was a strong correlation between non-HDL-c with both ApoB $(r=0.808)$ and oxidized LDL-c $(r=0.588)$.

Conclusions: These findings emphasize the limitations of only considering LDL-c concentration for cardiovascular (CV) risk assessment. Targeting only LDL-C could result in missed opportunities for $\mathrm{CV}$ risk reduction in T2DM patients. These data suggest that non-HDL-C, ApoB and oxidized LDL-c levels could be considered as an important part of these patients' evaluation allowing for a more accurate estimation of CV risk and hopefully better management of these high-risk patients.
\end{abstract}

Keywords: Dyslipidemia, ApoB, LDL-C, CVD, CV risk, Non-HDL cholesterol, T2DM

\footnotetext{
* Correspondence: lilianafonsecaa@gmail.com

${ }^{1}$ Endocrinology Department, Centro Hospitalar e Universitário do Porto, Largo Professor Abel Salazar, 4099-001 Porto, Portugal

Full list of author information is available at the end of the article
}

C C The Author(s). 2020 Open Access This article is licensed under a Creative Commons Attribution 4.0 International License, which permits use, sharing, adaptation, distribution and reproduction in any medium or format, as long as you give appropriate credit to the original author(s) and the source, provide a link to the Creative Commons licence, and indicate if changes were made. The images or other third party material in this article are included in the article's Creative Commons. licence, unless indicated otherwise in a credit line to the material. If material is not included in the article's Creative Commons licence and your intended use is not permitted by statutory regulation or exceeds the permitted use, you will need to obtain permission directly from the copyright holder. To view a copy of this licence, visit http://creativecommons.org/licenses/by/4.0/ The Creative Commons Public Domain Dedication waiver (http://creativecommons.org/publicdomain/zero/1.0/) applies to the data made available in this article, unless otherwise stated in a credit line to the data. 


\section{Background}

Diabetes mellitus is a risk factor for cardiovascular disease (CVD) and has been associated with 2- to 4-fold higher mortality [1-5]. In addition to glycemic control, the cornerstone of type 2 diabetes mellitus (T2DM) patients treatment is the management of the several cardiovascular risk factors that commonly are present in these patients. Dyslipidemia is common in T2DM patients and there is strong evidence that lowering cholesterol levels improves cardiovascular outcomes, even in patients with apparently adequate lipid profiles [6].

Low-density-lipoprotein cholesterol (LDL-c) is recommended as the primary target to guide lipid-lowering therapy [7]. However, there is evidence that other lipid parameters are also relevant in order to reduce coronary heart disease (CHD) events [8, 9], such as non-highdensity lipoprotein cholesterol (non-HDL-c) and Apolipoprotein B (ApoB) which were considered secondary targets for lipid-lowering therapy according to the 2016 European Society of Cardiology/European Atherosclerosis Society (ESC/EAS Guidelines) [7]. It has been suggested that non-HDL-c or ApoB may provide a more accurate estimation of CHD risk than LDL-c $[8,9]$. In 2019, the ESC/EAS Guidelines [5] recommended that, when evaluating patients with diabetes, metabolic syndrome, obesity, high triglyceride concentration or very low LDL-c levels, non-HDL and ApoB could be preferred in order to estimate CV risk. These guidelines also updated LDL-c targets and risk categories, classifying all T2DM patients with moderate, high or very-high $\mathrm{CV}$ risk.

T2DM patients present an atherogenic dyslipidemia characterized by increased triglycerides, decreased HDL particles, and LDL levels overlapping those of nondiabetics. Nevertheless, they also present small and dense LDL particles that are highly atherogenic and strongly increase these patients' CV risk. Oxidized lipids in circulating LDL-c are also shown to be strongly associated with coronary atherosclerosis, arterial dysfunction, and mortality [10-12]. Therefore, when evaluating a diabetic patient with a basic lipid profile, which usually determines LDL-c, total cholesterol, triglycerides and HDL-c levels, its severe atherogenic susceptibility might not be reflected since in many patients these markers remain within the normal range [7], and thus, CV risk can be underestimated.

The aim of this study was to compare a comprehensive lipid profile between patients with type 2 diabetes mellitus (T2DM) with LDL-c concentration within and above target it was, according to the 2016 and 2019 guidelines.

\section{Patients and methods}

A survey on our institution's electronic medical records for patients with an extended lipid profile collected at a Portuguese University Hospital was performed. Patients followed in the Dyslipidemia outpatient department between January 2010 and September 2017 were selected. After analysis of the electronic medical records of 345 patients, 96 T2DM patients were included in this study. This study was approved by the local Ethics committee (150-DEFI/149-CES). Due to the retrospective design of this study, consent to participate was waived by the Ethics Committee.

Diabetes mellitus was defined as plasma glucose $\geq 126$ $\mathrm{mg} / \mathrm{dL}$ in at least two measurements, glycated hemoglobin $(\mathrm{HbA} 1 \mathrm{c}) \geq 6.5 \%$ or prescription of any antidiabetic medication [13].

Patients were classified as smokers if they consumed at least 1 cigarette per week. A former smoker was defined as having quit smoking at least 6 months before assessment. Alcohol consumption was determined when patients reported drinking at least $10 \mathrm{~g}$ or $20 \mathrm{~g}$ of pure ethanol daily, for women and men respectively. Patients with an history of stroke and/or transient ischaemic attack were considered to have cerebrovascular disease.

Conditions that could interfere with the lipid profile such as genetic dyslipidemia, hepatic or renal moderate to severe disease, cancer, viral infection (hepatitis B or $\mathrm{C}$, human immunodeficiency virus), genetic metabolic disease, hyper or hypothyroidism were considered exclusion criteria. Pediatric patients and pregnant women were also excluded as well as patients treated with drugs that could alter the lipid profile as a side effect, such as glucocorticoids.

In order to stratify risk categories, patients were classified as having "very high risk" and "high risk" according to the 2016 ESC/EAS Guidelines for the Management of Dyslipidemias [7]. LDL-c targets defined by the 2016 ESC/EAS Guidelines [7] were as follows: LDL-c $<70 \mathrm{mg} / \mathrm{dL}$ for very high-risk patients and LDL-c $<100 \mathrm{mg} / \mathrm{dL}$ for high risk patients. The same guidelines were used to define the targets of the remaining lipoproteins [7]: ApoB: $<80 \mathrm{mg} / \mathrm{dL}$ in very high risk patients and $<100 \mathrm{mg} / \mathrm{dL}$ in high risk patients; non-HDL-c: < $100 \mathrm{mg} / \mathrm{dL}$ in very high risk patients and < $130 \mathrm{mg} / \mathrm{dL}$ in high risk patients; ApoA1: low if < $120 \mathrm{mg} / \mathrm{dL}$ in men and $<140 \mathrm{mg} / \mathrm{dL}$ in women; lipoprotein (a) $(\mathrm{Lp}(\mathrm{a}))<50 \mathrm{mg} / \mathrm{dL}$. For the remaining parameters, the following laboratory reference values were used: oxidized LDL-c: $26-117 \mathrm{mg} / \mathrm{dL}$ and ApoB/ApoA1 ratio: 0.45-1.25. In the 2019 guidelines update, risk categories were reviewed and new targets were defined according to patient's CV risk: $\mathrm{LDL}-\mathrm{c}<55$, $<70$ and $<100 \mathrm{mg} / \mathrm{dL}$ for very-high-, high-, and moderate-risk patients, respectively; $\mathrm{ApoB}<65$, 80 , and $100 \mathrm{mg} / \mathrm{dL}$ for very-high-, high-, and moderate-risk patients, respectively; and, non-HDL-c $<85,100$, and 130 $\mathrm{mg} / \mathrm{dL}$ for very-high-, high-, and moderate-risk patients, respectively.

Biochemical laboratory tests were conducted after an 8-h night fast. Lipid parameters included total cholesterol, 
HDL-c, triglycerides, ApoB, ApoA1, oxidized LDL-c and Lp(a). LDL-c levels were calculated through Friedewald's formula [14]: LDL-c $(\mathrm{mg} / \mathrm{dL})=$ total cholesterol $(\mathrm{mg} / \mathrm{dL})-$ HDL-c $(\mathrm{mg} / \mathrm{dL})$ - triglycerides $(\mathrm{mg} / \mathrm{dL}) / 5$, unless triglycerides $\geq 400 \mathrm{mg} / \mathrm{dL}$, in which case direct LDL-c measurement was performed. Non-HDL-c was calculated by subtracting HDL-c to total cholesterol. Other parameters such as creatinine, uric acid, glucose and homocysteine were also evaluated. HbA1c was assessed in most diabetic patients. Creatinine, glucose, uric acid, total cholesterol, HDL-c and triglycerides were measured using an enzymatic colorimetric method, with intra and inter-assay coefficients of variation of $<2.8 \%$ and $<3.9 \%$, respectively. These parameters were measured using an automated autoanalyzer (Cobas 8000, Roche Diagnostics, Mannheim, Germany). ApoA1, ApoB and Lp(a) were evaluated through immunoturbidimetry, with intra and inter-assay coefficients of variation of $<1 \%$ and $<2.4 \%$ for ApoA $1,<1.2 \%$ and $<3.2 \%$ for ApoB and $<3.7 \%$ and $<3.8 \%$ for $\mathrm{Lp}(\mathrm{a})$, respectively. These parameters were evaluated using an automated autoanalyzer (Cobas Integra 400, Roche Diagnostics, Mannheim, Germany). Oxidized LDL-c was measured by Sandwich Enzyme-Linked ImmunoSorbent Assay (Mercodia, Uppsala, Sweden), with intra and inter-assay coefficients of variation of $<7.3 \%$ and $<8.3 \%$, respectively. Small and dense low-density lipoprotein-cholesterol (sd-LDL) particles were measured using an enzymatic colorimetric method (Randox Laboratories, UK) with intra and inter-assay coefficients of variation of $<3 \%$. HbA1c was determined using an ionexchange chromatography method (Variant II turbo, BioRad Laboratories, CA, USA), with intra and inter-assay coefficients of variation of $<0.78 \%$ and $<0.66 \%$, respectively. Homocysteine was measured by nephelometry (Dimension Vista 500, Siemens Healthcare, Germany), with intra and inter-assay coefficients of variation of $<3.3 \%$ and $<8.2 \%$, respectively.

\section{Statistical analysis}

Statistical analysis was performed using IBM SPSS ${ }^{\bullet}$ version 21.0 and a $p$ value below 0.05 was considered statistically significant. For continuous variables, distribution normality was tested through histogram observation, kurtosis and skewness analysis. Results are presented as mean value \pm standard-deviation and median values (25-75 percentiles). The chi-square test and the Fisher's exact test were applied to analyze differences between groups regarding categorical variables. The Student $t$ test for independent variables and the Mann Whitney test were used to compare continuous variables with normal and non-normal distribution between groups, respectively. Correlations were evaluated using the Pearson and the Spearman correlation test for continuous symmetrical and asymmetrical variables, respectively.

\section{Results}

This study evaluated 96 patients with T2DM (56 men and 40 women) with a mean age of $58.9 \pm 9.0$ years, median diabetes duration of 10 years (IQR: 4-7) and mean HbA1c of $8.1 \pm 1.9 \%$. The characterization of the sample is presented in Table 1. Mean LDL-c levels were 102.4 \pm $38.6 \mathrm{mg} / \mathrm{dL}$. The majority of patients $(n=48 / 72,66.7 \%)$ were under lipid-lowering drugs and statins were the most common ( $n=44 / 72,45.8 \%), 90.4 \%$ (40/44) under moderate-intensity statins and the others under highintensity statins. Ten $(13.9 \%)$ patients were on treatment with fibrates and 4 (5.6\%) with ezetimibe. Patients were not taking other drugs for treating dyslipidemia or any anti-diabetic drugs that could modify the lipid profile such as thiazolidinediones, glucagon-like peptide-1 agonists or sodium-glucose co-transporter-2 inhibitors. Most patients were under treatment with metformin (71.9\%), followed by gliptins (53.1\%), insulin (47.9\%) and sulfonylureas (20.8\%). According to the ESC/EAS guidelines, patients were divided in two groups: patients with LDL-c levels within target $(n=27)$ and LDL-c levels above target $(n=69)$ (Table 1$)$.

There were no statistically significant differences between the groups regarding gender, age, body mass index (BMI), waist circumference, HbA1c, triglycerides, HDL-c or glucose levels. No statistically significant differences between groups were found in relation to alcohol ingestion, smoking or use of lipid lowering therapy. Uric acid and homocysteine levels, predictors of new CV events, were not statistically different between groups. Patients with LDL-c levels above target had higher hypertension prevalence, although no significant difference was found when comparing the measurements of systolic and diastolic blood pressure.

The lipid profile of T2DM patients with LDL-c within and above target is presented in Table 2. Mean LDL-c concentration was $64.8 \pm 14.7 \mathrm{mg} / \mathrm{dL}$ in patients with LDL-c within target and $117.1 \pm 34.9 \mathrm{mg} / \mathrm{dL}$ in those with LDL-c above target $(p<0.001)$. The latter group also presented statistically significant higher total cholesterol, ApoB, non-HDL-c, ApoB/ApoA1 ratio and oxidized LDL-c levels. No statistically significant differences were found between the two groups regarding $L p(a)$ and ApoA1 particles. Despite having an LDL-c within the target defined by the 2016 guidelines, these patients presented elevated levels of other atherogenic lipoproteins. In fact, 22.2, 25 and $44.4 \%$ of patients presented nonHDL-c, ApoB and oxidized LDL-c levels, respectively, above the threshold for CV risk.

After reclassification of the targets according to the 2019 guidelines, 16 (16.7\%) patients were regarded has having LDL-c within target. Still, in these patients, 50, 38.8 and $43.8 \%$ presented elevated non-HDL-c, ApoB and oxidized-LDL-c, respectively. Of note, in patients 
Table 1 Comparison of clinical and laboratory variables between type 2 diabetes patients with LDL-c within and above target

\begin{tabular}{|c|c|c|c|c|c|}
\hline & $\mathrm{n}$ & LDL-c within target $(\boldsymbol{n}=27)$ & $\mathrm{n}$ & LDL-c above target $(\boldsymbol{n}=69)$ & $p$ \\
\hline Male n (\%) & 27 & $16(59.3 \%)$ & 69 & $40(58.0 \%)$ & 0.908 \\
\hline Age (years) & 27 & $59.8 \pm 9.7$ & 69 & $58.6 \pm 8.8$ & 0.571 \\
\hline Duration of diabetes* (years) & 22 & $10.0(3.5-15.3)$ & 55 & $10.0(5.0-19.0)$ & 0.412 \\
\hline Cardiovascular disease $n(\%)$ & 26 & 7 (26.9\%) & 68 & $15(22.1 \%)$ & 0.618 \\
\hline Cerebrovascular disease n (\%) & 26 & $5(7.4 \%)$ & 68 & $4(15.4 \%)$ & $0.256^{1}$ \\
\hline Peripheral vascular disease $\mathrm{n}(\%)$ & 27 & $5(7.4 \%)$ & 68 & $2(7.7 \%)$ & $0.955^{1}$ \\
\hline Body mass index $\left(\mathrm{Kg} / \mathrm{m}^{2}\right)$ & 20 & $29.9 \pm 3.7$ & 56 & $30.6 \pm 4.8$ & 0.553 \\
\hline Systolic BP (mmHg) & 24 & $142.0 \pm 20.9$ & 59 & $141.5 \pm 18.3$ & 0.928 \\
\hline Diastolic BP (mmHg) & 11 & $71.8 \pm 12.0$ & 26 & $78.2 \pm 15.4$ & 0.229 \\
\hline Waist circumference $(\mathbf{M})(\mathrm{cm})$ & 12 & $102.1 \pm 9.5$ & 17 & $105.0 \pm 11.9$ & 0.492 \\
\hline Waist circumference $(\mathrm{F})(\mathrm{cm})$ & 3 & $96.0 \pm 13.4$ & 15 & $98.0 \pm 12.7$ & 0.438 \\
\hline Triglycerides* (mg/dL) & 27 & $146.0(106.0-295.0)$ & 69 & $146.0(108.0-207.5)$ & 0.300 \\
\hline HDL-c (M) (mg/dL) & 16 & $35.2 \pm 9.68$ & 40 & $41.7 \pm 12.4$ & 0.064 \\
\hline HDL-c (F) (mg/dL) & 11 & $50.5 \pm 10.7$ & 29 & $46.6 \pm 10.2$ & 0.301 \\
\hline Glucose* (mg/dL) & 22 & $137.0(108.0-182.3)$ & 59 & 151.5 (119.0-226.8) & 0.062 \\
\hline HbA1c (\%) & 18 & $7.8 \pm 2.6$ & 48 & $8.2 \pm 1.6$ & 0.538 \\
\hline Uric acid (mg/dL) & 21 & $5.1 \pm 0.4$ & 44 & $4.9 \pm 0.2$ & 0.656 \\
\hline Homocysteine ( $\mu \mathrm{mol} / \mathrm{L})$ & 20 & $11.4 \pm 1.2$ & 55 & $12.2 \pm 0.8$ & 0.632 \\
\hline Statin use $n(\%)$ & 20 & $14(70.0 \%)$ & 52 & $30(57.7 \%)$ & 0.337 \\
\hline Fibrates use n (\%) & 20 & $2(10 \%)$ & 52 & $8(15.4 \%)$ & $0.716^{1}$ \\
\hline Ezetimibe use $\mathrm{n}(\%)$ & 20 & $3(15 \%)$ & 51 & $1(2 \%)$ & $0.065^{1}$ \\
\hline Alcohol drinking n (\%) & 8 & $1(12.5 \%)$ & 20 & 7 (35.0\%) & $0.371^{1}$ \\
\hline Smoking n (\%) & 21 & $2(9.5 \%)$ & 61 & $7(11.5 \%)$ & $0.805^{1}$ \\
\hline Hypertension n (\%) & 27 & $14(51.9 \%)$ & 68 & $56(82.4 \%)$ & 0.020 \\
\hline
\end{tabular}

Data are presented as mean \pm standard deviation, unless otherwise indicated by $*$ corresponding to data presented as median, 25 th and 75 th percentiles and ${ }^{1}$ corresponding Fisher's exact test. $L D L-c$ Low-density lipoprotein cholesterol, BP Blood pressure, $H D L-c$ High-density lipoprotein cholesterol, $M$ male, $F$ female

with ApoB within target, 16.7\% had elevated LDL-c. The lipid profile of the patients is exhibited in Table 3.

LDL-c exhibited positive correlations with several lipid parameters. After Bonferroni correction, statistically significant positive correlations between LDL-c and total cholesterol $(r=0.895, p<0.001)$, non-HDL-c $(r=0.850$, $p<0.001)$, ApoB $(r=0.656, p<0.001)$, ApoB/ApoA1 ratio $(r=0.291, p=0.008)$ and oxidized-LDL-c $(r=0.508$, $p<0.001)$ were found. Non-HDL-c was also significantly and positively correlated with $\mathrm{ApoB}(r=0.808, p<0.001)$ and oxidized-LDL-c $(r=0.588, p<0.001)$.

Table 2 Comparison of lipid profile between type 2 diabetes patients with LDL-c within and above target (2016 guidelines criteria)

\begin{tabular}{|c|c|c|c|c|c|}
\hline & $\mathrm{n}$ & LDL-c within target & $n$ & LDL-c above target & $p$ \\
\hline LDL-c (mg/dL) & 27 & $65(53-70)$ & 69 & $109.0(92.5-133.0)$ & $<0.001$ \\
\hline Total cholesterol (mg/dL) & 27 & $146(125-162)$ & 69 & $180.0(74.3-107.8)$ & $<0.001$ \\
\hline ApoB (mg/dL) & 24 & $67.0(58.8-79.0)$ & 64 & $85.0(74.3-107.8)$ & $<0.001$ \\
\hline ApoA1 (F) (mg/dL) & 8 & 167.5 (123.0-193.5) & 27 & $155(140-180)$ & 0.556 \\
\hline ApoA1 (M) (mg/dL) & 15 & $130(102-143)$ & 33 & 134.0 (116.5-139.5) & 0.772 \\
\hline ApoB/ApoA1 ratio & 22 & $0.54(0.41-0.64)$ & 60 & $0.63(0.54-0.76)$ & 0.004 \\
\hline Non-HDL-c* (mg/dL) & 27 & $99.0(85.0-118.0)$ & 69 & $142.2(119.5-166.5)$ & $<0.001$ \\
\hline Oxidized LDL-c* (U/L) & 27 & $115.7(94.5-162.1)$ & 69 & $150.8(117.3-193.4)$ & 0.007 \\
\hline Lipoprotein $(a)^{*}(m g / d L)$ & 22 & $9.5(4.0-34.5)$ & 62 & $25(5.75-58.10)$ & 0.106 \\
\hline
\end{tabular}


Table 3 Comparison of lipid profile between type 2 diabetes patients with LDL-c within and above target - reclassification according to the 2019 guidelines

\begin{tabular}{|c|c|c|c|c|c|}
\hline & $n$ & LDL-c within target & $n$ & LDL-c above target & $p$ \\
\hline LDL-c (mg/dL) & 16 & $55.5(49-66)$ & 80 & $104.5(85.3-128.8)$ & $<0.001$ \\
\hline Total cholesterol $(\mathrm{mg} / \mathrm{dL})$ & 16 & $143(125.8-150.8)$ & 80 & $177(159-213)$ & $<0.001$ \\
\hline ApoB (mg/dL) & 13 & $70(54.5-86.5)$ & 75 & $83(70-106)$ & 0.037 \\
\hline ApoA1 (F) (mg/dL) & 3 & $160(N A)$ & 32 & 157.5 (140.3-180.8) & 0.976 \\
\hline ApoA1 (M) (mg/dL) & 9 & $135(107.5-145.0)$ & 39 & $134(115-140)$ & 0.792 \\
\hline ApoB/ApoA1 ratio & 12 & $0.56(0.39-0.68)$ & 70 & $0.60(0.51-0.74)$ & 0.172 \\
\hline Non-HDL-c* (mg/dL) & 16 & $95.5(84.3-117.3)$ & 80 & $138(115.0-164.8)$ & $<0.001$ \\
\hline Oxidized LDL-c* (U/L) & 16 & $113.8(76.2-160.2)$ & 80 & $148(113.9-185.5)$ & 0.024 \\
\hline Lipoprotein $(a)^{*}(\mathrm{mg} / \mathrm{dL})$ & 10 & $6(4.0-25.8)$ & 74 & $24.5(5.0-53.5)$ & 0.232 \\
\hline
\end{tabular}

Data are presented as mean \pm standard deviation, unless otherwise indicated by $*$ corresponding to data presented as median, 25 th and 75 th percentiles. $L D L-c$ Low-density lipoprotein cholesterol, $A p o B$ Apolipoprotein B, ApoA1 Apolipoprotein A1, NA not applicable, $M$ male, F female, Non-HDL-c non-high-density lipoprotein cholesterol

\section{Discussion}

Cardiovascular disease is the leading cause of death in patients with diabetes [15], making cardiovascular risk reduction a priority in these patients. LDL-c is recommended as a primary target to reduce $\mathrm{CV}$ risk by the 2016 and 2019 ESC/EAS Guidelines [7]. Nonetheless, even T2DM patients with LDL-c within the target range still have CV events, indicating there is residual CV risk that is unaccounted by LDL-c assessment. In accordance, the 2019 guidelines update have also highlighted the importance of ApoB and non-HDL-c determination in patients with diabetes. In fact, ApoB is also recommended to assess CV risk, particularly in patients with hypertriglyceridemia, diabetes, obesity, metabolic syndrome and very low LDL-c levels, and it can be used as an alternative to LDL-c for screening, diagnosis and management $[5,16]$. The present study shows that in T2DM patients there are several atherogenic lipoproteins that remain elevated even in patients presenting an adequate LDL-c level. This study evidenced that 25 and 22.2\% of T2DM patients had high ApoB and non-HDL-C levels, respectively, regardless of presenting an LDL-c concentration below the threshold for which treatment initiation is recommended. Moreover, when applying the LDL-c targets proposed by the 2019 guidelines, 38.8 and $50 \%$ of patients presented ApoB and non-HDL-c levels above target, respectively. Thus, as suggested by the 2019 guidelines, in patients with diabetes, LDL-c concentration seems to be insufficient to estimate the real $\mathrm{CV}$ risk. In this study, individuals with LDL-c levels above target had a higher rate of hypertension. Some studies have demonstrated that, when improving LDL-c and serum uric acid levels, pharmacologically or through lifestyle modification, the risk of developing hypertension is reduced $[17,18]$. Cicero et al. demonstrated that in an overall healthy population sample, the contemporary presence of suboptimal serum uric acid and LDL-c values were associated with an increased risk of developing hypertension [17].

\section{ApoB and non-HDL}

ApoB and non-HDL are not routinely determined, since they are considered secondary targets [7]. However, several studies have demonstrated an important role of both non-HDL and ApoB, when compared with LDL-c, regarding the occurrence of CHD events $[19,20]$. In fact, ApoB levels have been shown to be associated with both arterial stiffness and cardiovascular disease [5, 21], whereas Cicero et al. demonstrated that ApoB levels were significantly associated with the carotid - femoral pulse wave velocity [22]. There is evidence that nonHDL cholesterol and ApoB may even be better predictors of CVD incidence among diabetic men than LDL-c [23]. This may occur because all major atherogenic particles originated in liver, namely, very-low density lipoproteins (VLDL) and intermediate-density lipoproteins have a apolipoprotein B100 molecule [24]. Therefore, ApoB measurement might be a direct proxy when assessing the number of atherogenic particles (LDL and nonLDL). Non-HDL-c has also been suggested as a better marker of CVD risk and coronary atherosclerosis [25]. Moreover, non-HDL-c seems to be associated with atherogenic dyslipidemia, insulin resistance, portal hyperinsulinemia and the metabolic syndrome phenotype [20, 24, 26-30]. Patients with higher triglyceride levels were shown to have lower LDL-c levels for any given ApoB concentration compared with subjects with lower triglyceride levels by Leroux et al. [31]. Thus, patients with hypertriglyceridemia, which is very frequent in T2DM patients, may present falsely lower LDL-c concentrations. However, these patients still have a high atherogenic risk, since ApoB levels remain elevated, even with decreased LDL-c levels [31]. Friedewald et al. recognized in their original work that, at lower LDL-c 
levels, even small errors in very low-density lipoprotein cholesterol estimation resulted in significant errors in LDL-c estimation [14] thus, in patients with LDL-c deemed appropriate by recent guidelines, the atherogenicity could be underestimated if only LDL-c is taken into account. To overcome this, the 2019 guidelines reinforce that, in patients with very low LDL-c levels, ApoB might be superior to LDL-c in assessing CV risk and guiding treatment. In this study, 22 and $25 \%$ of T2DM patients had high non-HDL-c and ApoB levels, respectively, even when presenting LDL-c within target. When analyzing data using the 2019 guidelines targets, the proportion of patients with elevated ApoB and non-HDL-c increased, despite having a LDL-c within target. These results suggest that in T2DM patients there is unaccounted CV risk if only LDL-c is considered, resulting in possible under treatment and missed CV benefits. This effect might be even more pronounced for lower LDL-c levels, in which the LDL-c/ApoB mismatch increases. Of note, in this study a number of patients (16.7\%) with ApoB within target, according to the 2019 guidelines, still presented elevated LDL-c.

\section{HDL-c and ApoA1}

Although an association between low HDL-c concentrations and an increased risk of T2DM has been shown at an epidemiological level [32, 33], serum HDL-c levels do not predict cardiometabolic risk, especially in T2DM [34]. HDL-c has a cardioprotective effect that has been attributed to its role in reverse cholesterol transport, its effects on endothelial cells, and antioxidant activity [35]. Similarly, HDL molecules also present antiinflammatory, anti-apoptotic, vasodilatory, antithrombotic, and anti-infectious properties and can modulate the glucose metabolism directly [36]. Despite those observations, increasing HDL-c levels through medication have not shown to reduce CV events and HDL isn't a primary target for $\mathrm{CV}$ risk reduction. In T2DM patients, HDL particles' functions are impaired [37-40] and ApoA1 catabolism, a major HDL particle protein, is increased [37, 41]. In fact, Watts et al. [42] observed that ApoA1 production was increased in these patients in order to match the increased clearance. The mechanisms of increased ApoA1 clearance in T2DM are largely unknown, however the increased triglycerides fraction on HDL and the reduced adiponectin plasma levels observed in T2DM patients have been suggested to accelerate ApoA1 degradation [43]. It is also unclear whether hyperglycemia itself is linked to the increased clearance and may contribute to impaired HDL function, since lipoproteins are the primary targets of hyperglycemiainduced nonenzymatic glycation. In fact, glycated ApoA1 isolated from T2DM patients shows impaired selfassociation and lipid-binding abilities, as well as reduced stability [37]. In vitro studies demonstrated that human HDL combination with glucose resulted in reduced cholesterol efflux capacity [37, 38]. Sangeeta et al. [37] studied a T2DM patients cohort and demonstrated that despite normal HDL-c concentration, ApoA1 metabolism and function were clearly abnormal. T2DM patients had increased ApoA1 turnover and impaired cholesterol efflux and antioxidant activity of HDL. In summary, these findings suggest that serum HDL-c level is not a marker of reverse cholesterol transport, and neither HDL-c nor ApoA1 levels reflect HDL-c functionality $[37,38]$. No differences were found in HDL-c levels between patients with LDL-c concentration within and above target. Low levels of ApoA1 also seem to independently associate with new onset of T2DM [44]. Nonetheless, in this study, patients evidenced normal ApoA1 concentrations and no differences were found between groups.

\section{ApoB/ApoA1 ratio}

ApoB/ApoA1 ratio is a simple and accurate measurement to estimate CVD risk [45, 46]. Carnevale et al. (2011) described that a low ApoB/ApoA1 ratio reflects a less atherogenic lipid profile, regardless of LDL-c [46]. Several studies have also suggested that an elevated ApoB/ApoA1 ratio is a more powerful predictor than other lipid fractions for metabolic disorders, including T2DM [47-52]. Recently, it has been demonstrated that ApoB/ApoA1 ratio is independently associated with carotid atherosclerosis in T2DM patients with controlled LDL-c levels [53]. In this study, both groups presented a mean $A p o B / A p o A 1$ ratio within the reference range. Park et al. [54] demonstrated that metabolic syndrome (MetS) patients showed an increased ApoA1 and suggested this was correlated with the presence of hypertriglyceridemia. Since T2DM patients frequently exhibited higher triglyceride levels, this could cause an increase in ApoA1 levels, decreasing the ApoB/ApoA1 ratio to normal range [54]. Patients with LDL-c levels above target, according to the 2016 guidelines, showed a significantly higher ratio than those within target. This observation didn't hold when using the 2019 guidelines as reference, suggesting that, by lowering LDL-c concentration even more, there is a decrease in ApoB particles capable of reducing this ratio. Further studies with a higher number of patients are necessary to test this hypothesis.

\section{Oxidized LDL-c}

In this study, $44.4 \%$ of T2DM patients and LDL-c within target had oxidized LDL-c above the reference range. Using the 2019 guidelines as reference, oxidized LDL-c concentration remained above the reference range in $43.8 \%$ of patients. Oxidized LDL-c has been associated with progression of atherosclerosis and CHD [55-57]. 
Holvoet et al. suggested that the predictive value of oxidized LDL-c seems to be additive to that of the Framingham global risk assessment score for CV risk [55]. Chronic hyperglycemia triggers the production of excess free radicals causing lipid molecules peroxidation in a chain reaction [58]. Malondialdephyde (MDA), oxidized LDL, oxidized LDL/LDL and oxidized LDL/HDL-c levels have been shown to be significantly elevated in T2DDM patients versus controls in several studies [59-61]. MDA is a recognized marker of lipid peroxidation and has been reported to modify ApoB, increasing the susceptibility of LDL-c to oxidation [56, 62]. Cicero et al. demonstrated a strong association of serum uric acid and oxidized LDL-c with carotid - femoral pulse wave velocity in individuals with normal and mildly impaired renal function. They suggested that serum uric acid and oxidized LDL-c should be monitored and managed as a marker of vessel stiffness [22]. In this study, even patients presenting LDL-c concentration within target showed elevated oxidized LDL-c levels, suggesting a high risk of atherosclerotic disease progression. This observation was maintained even after lowering the LDL-c targets according to the 2019 guidelines, indicating that oxidized LDL-c levels are not affected by LDL-c levels and could be a reliable index of atherogenicity in T2DM patients, regardless of the LDL-c status.

\section{Lipid parameters correlations}

In T2DM dyslipidemia presents with a high concentration of free fatty acids and triglycerides, low HDL-c levels and increased sd-LDL particles and ApoB [63]. In this study LDL-c was positively correlated with total cholesterol, non-HDL-c and ApoB. Non-HDL also demonstrated a high correlation with ApoB and oxidized LDL-C, even stronger than for LDL-c. While ApoB and oxidized LDL-c assessment may not be routinely available, non-HDL-c measurement, as it is inexpensive and easy to obtain, could have a major role on identifying an atherogenic profile [29]. Additionally, non-HDL-c seems able to predict CVD over a wider range of triglyceride concentrations [29] surpassing Friedewald's formula limitation, which is affected by triglycerides concentrations.

\section{Strength and study limitations}

This study has some limitations that deserve comment. First, this is a small sample, especially regarding female gender. Secondly, it was a cross-sectional study with an associated bias not susceptible to rule out, especially the non-assessment of long-term outcomes, such as the occurrence of CVD according to lipid levels parameters. Nevertheless, these results can motivate a prospective study to evaluate if reducing other atherogenic lipoproteins, along with LDL-c, results in a greater reduction of $\mathrm{CV}$ events and mortality. This study also has strengths.
A small number of studies have assessed nonconventional lipid parameters such as ApoA1, ApoB, Lp(a) or oxidized LDL-c in T2DM patients. Moreover, it is an important contribution to the characterization of a comprehensive lipid profile in patients with this condition. Finally, this study has important clinical implications since it confirms that, in T2DM, assessing solely LDL-c is insufficient for the correct estimation of the $\mathrm{CV}$ risk, resulting in a suboptimal treatment of these patients. There was a high mismatch LDL-c/ApoB thus, reinforcing the ESC/EAS 2019 guidelines recommendation of including ApoB measurement in patients with T2DM in order to better evaluate their atherogenic risk and guide adjustment of lipid-lowering therapy after achievement of the recommended LDL-c goal. Since a few patients with ApoB within target presented high LDL-c, LDL-C and ApoB should be additive regarding CV risk evaluation.

\section{Conclusions}

LDL-c levels are currently accepted as the main therapeutic target for the prevention of $\mathrm{CV}$ events in T2DM patients. Nevertheless, even in patients with LDL-c considered within target, some patients still present atherosclerosis progression and CVD-related events. This study evidences that, although having LDL-c within target, patients presented elevated levels of molecules known for their atherogenic character, namely oxidized LDL-c, non- HDL-c and ApoB. The present study contributes to a better understanding of lipid metabolism in T2DM patients and has important clinical implications since it suggests that LDL-c seems to be insufficient to estimate CV risk. These data suggest that combining LDL-c with ApoB, oxidized LDL-c and non-HDL-c may add important information in order to better estimate CV risk in individuals with T2DM. Further studies should evaluate if intensive therapy, not only to reduce LDL-c but also other lipoproteins such as non-HDL-c, ApoB or oxidized LDL-c, has a beneficial impact on CVD and mortality.

\footnotetext{
Abbreviations

ApoA1: Apolipoprotein A1; ApoB: Apolipoprotein B; BP: Blood pressure; CHD: Coronary heart disease; CV: Cardiovascular; CVD: Cardiovascular disease; EAS: European Atherosclerosis Society; ESC: European Society of Cardiology; F: Female; HbA1c: Glycated hemoglobin; HDL-c: High-density lipoprotein cholesterol; LDL-c: Low-density lipoprotein cholesterol; Lp(a): Lipoprotein (a); M: Male; MetS: Metabolic Syndrome; Non-HDL-c: Non-high-density lipoprotein cholesterol; SCORE: Systemic Coronary Risk Estimation; SD: Standard deviation; sd-LDL-c: Small and dense low-density lipoproteincholesterol; T2DM: Type 2 diabetes mellitus; VLDL: Very-low density lipoproteins
}

Acknowledgements None. 


\section{Authors' contributions}

L.F., S.P., H.R., J.C.O. and I. P designed the study; L.F. and S.P. acquired the data; S.P. and L.F. did the data analysis; L.F., S. P, H.R., J.C.O. and I.P. interpreted the data; L.F. and S.P. drafted the work and all authors revised it critically for important intellectual content. All authors approved the final version submitted and are accountable for all aspects of the work. All authors read and approved the final manuscript.

\section{Funding}

No funding was received for this study.

\section{Availability of data and materials}

The datasets generated during and/or analysed during the current study are available from the corresponding author on reasonable request.

\section{Ethics approval and consent to participate}

All procedures performed in studies involving human participants were in accordance with the ethical standards of the institutional and/or national research committee and with the 1964 Helsinki declaration and its later amendments or comparable ethical standards. This study was approved by the local Ethics committee (150-DEFI/149-CES). Due to the retrospective nature of this study, consent to participate was waived by the Ethics Committee.

\section{Consent for publication}

Liliana Fonseca assigns Lipids in Health and Disease all rights of copyright.

\section{Competing interests}

The authors declare that they have no competing interests. Liliana Fonseca declares that she has no conflict of interest. Silvia Paredes declares that she has no conflict of interest. Helena Ramos declares that she has no conflict of interest. José Carlos Oliveira declares that he has no conflict of interest. Isabel Palma declares that she has no conflict of interest.

\section{Author details}

${ }^{1}$ Endocrinology Department, Centro Hospitalar e Universitário do Porto, Largo Professor Abel Salazar, 4099-001 Porto, Portugal. Endocrinology Department, Hospital de Braga, Sete Fontes, São Victor, 4710-243 Braga, Portugal. ${ }^{3}$ Clinical Chemistry Department, Centro Hospitalar e Universitário do Porto, Largo Professor Abel Salazar, 4099-001 Porto, Portugal.

Received: 8 February 2020 Accepted: 20 May 2020

Published online: 06 June 2020

\section{References}

1. Einarson TR, Acs A, Ludwig C, Panton UH. Prevalence of cardiovascular disease in type 2 diabetes: a systematic literature review of scientific evidence from across the world in 2007-2017. Cardiovasc Diabetol. 2018;17:83.

2. Association AD. 10. Cardiovascular disease and risk management: standards of medical Care in Diabetes-2020. Diabetes Care. 2020;43:S111-34.

3. Strain WD, Paldanius P. Diabetes, cardiovascular disease and the microcirculation. Cardiovasc Diabetol. 2018;17:57.

4. Raghavan S, Vassy JL, Ho YL, Song RJ, Gagnon DR, Cho K, Wilson PW, Phillips LS. Diabetes mellitus-related all-cause and cardiovascular mortality in a National Cohort of adults. J Am Heart Assoc. 2019;8:e011295.

5. Mach F, Baigent C, Catapano AL, Koskina KC, Casula M, Badimon L, Chapman MJ, De Backer GG, Delgado V, Ference BA. 2019 ESC/EAS guidelines for the management of dyslipidaemias: lipid modification to reduce cardiovascular risk. Eur Heart J. 2020;41(1):111-8.

6. Schofield JD, Liu Y, Rao-Balakrishna P, Malik RA, Soran H. Diabetes dyslipidemia. Diabetes Therapy. 2016;7:203-19.

7. Catapano AL, Graham I, De Backer G, Wiklund O, Chapman MJ, Drexel H, Hoes AW, Jennings CS, Landmesser U, Pedersen TR. 2016 ESC/EAS guidelines for the management of dyslipidaemias. Eur Heart J. 2016;37: 2999-3058.

8. Lim Y, Yoo S, Lee SA, Chin SO, Heo D, Moon JC, Moon S, Boo K, Kim ST, Seo HM. Apolipoprotein B is related to metabolic syndrome independently of low density lipoprotein cholesterol in patients with type 2 diabetes. Endocrinol Metab. 2015;30:208-15.
9. Kilgore M, Muntner P, Woolley JM, Sharma P, Bittner V, Rosenson RS. Discordance between high non-HDL cholesterol and high LDL-cholesterol among US adults. J Clin Lipidol. 2014;8:86-93.

10. Toikka JO, Niemi P, Ahotupa M, Niinikoski H, Viikari JS, Rönnemaa T, Hartiala $\mathrm{JJ}$, Raitakari OT. Large-artery elastic properties in young men: relationships to serum lipoproteins and oxidized low-density lipoproteins. Arterioscler Thromb Vasc Biol. 1999;19:436-41.

11. Linna M, Ahotupa M, Löppönen MK, Irjala K, Vasankari T. Circulating oxidised LDL lipids, when proportioned to HDL-c, emerged as a risk factor of allcause mortality in a population-based survival study. Age Ageing. 2012;42: $110-3$.

12. Tiainen S, Kiviniemi A, Hautala A, Huikuri H, Ukkola O, Tokola K, Tulppo M, Vasankari T. Effects of a two-year home-based exercise training program on oxidized LDL and HDL lipids in coronary artery disease patients with and without Type-2 diabetes. Antioxidants. 2018;7:144.

13. Association AD. 2. Classification and diagnosis of diabetes: standards of medical care in diabetes_-2019. Diabetes Care. 2019;42:S13-28.

14. Friedewald WT, Levy RI, Fredrickson DS. Estimation of the concentration of low-density lipoprotein cholesterol in plasma, without use of the preparative ultracentrifuge. Clin Chem. 1972;18:499-502.

15. Balakumar $P$, Maung- $U$ K, Jagadeesh G. Prevalence and prevention of cardiovascular disease and diabetes mellitus. Pharmacol Res. 2016;113: 600-9.

16. Paredes S, Fonseca L, Ribeiro L, Ramos H, Oliveira JC, Palma I. Novel and traditional lipid profiles in metabolic syndrome reveal a high atherogenicity. Sci Rep. 2019;9:1-7.

17. Cicero AF, Fogacci F, Giovannini M, Grandi E, D'Addato S, Borghi C, Group BHS. Interaction between low-density lipoprotein-cholesterolaemia, serum uric level and incident hypertension: data from the Brisighella heart study. J Hypertens. 2019;37:728-31.

18. Cicero AF, Rosticci M, Bove M, Fogacci F, Giovannini M, Urso R, D'Addato S, Borghi C, Group BHS. Serum uric acid change and modification of blood pressure and fasting plasma glucose in an overall healthy population sample: data from the Brisighella heart study. Ann Med. 2017:49:275-82.

19. Boekholdt SM, Arsenault BJ, Mora S, Pedersen TR, LaRosa JC, Nestel PJ, Simes RJ, Durrington P, Hitman GA, Welch K. Association of LDL cholesterol, non-HDL cholesterol, and apolipoprotein B levels with risk of cardiovascular events among patients treated with statins: a meta-analysis. JAMA. 2012; 307:1302-9.

20. Sniderman AD, Williams K, Contois JH, Monroe HM, McQueen MJ, de Graaf J, Furberg CD. A meta-analysis of low-density lipoprotein cholesterol, nonhigh-density lipoprotein cholesterol, and apolipoprotein B as markers of cardiovascular risk. Cir Cardiovasc Qual Outcomes. 2011;4:337-45.

21. Holewijn S, Den Heijer M, Swinkels DW, Stalenhoef AH, De Graaf J. Apolipoprotein B, non-HDL cholesterol and LDL cholesterol for identifying individuals at increased cardiovascular risk. J Intern Med. 2010;268:567-77.

22. Cicero AF, Kuwabara M, Johnson R, Bove M, Fogacci F, Rosticci M, Giovannini M, D'Addato S, Borghi C, group BHS. LDL-oxidation, serum uric acid, kidney function and pulse-wave velocity: data from the Brisighella heart study cohort. Int J Cardiol. 2018;261:204-8.

23. Jiang R, Schulze MB, Li T, Rifai N, Stampfer MJ, Rimm EB, Hu FB. Non-HDL cholesterol and apolipoprotein B predict cardiovascular disease events among men with type 2 diabetes. Diabetes Care. 2004:27:1991-7.

24. Hermans MP, Sacks FM, Ahn SA, Rousseau MF. Non-HDL-cholesterol as valid surrogate to apolipoprotein B 100 measurement in diabetes: discriminant ratio and unbiased equivalence. Cardiovasc Diabetol. 2011;10:20.

25. Zhang Y, Wu N-Q, Li S, Zhu C-G, Guo Y-L, Qing P, Gao Y, Li X-L, Liu G, Dong Q. Non-HDL-C is a better predictor for the severity of coronary atherosclerosis compared with LDL-C. Heart Lung Circ. 2016;25:975-81.

26. Sniderman AD, St-Pierre AC, Cantin B, Dagenais GR, Després J-P, Lamarche B. Concordance/discordance between plasma apolipoprotein B levels and the cholesterol indexes of atherosclerotic risk. Am J Cardiol. 2003;91:1173-7.

27. Wägner AM, Pérez A, Zapico E, Ordóñez-Llanos J. Non-HDL cholesterol and apolipoprotein B in the dyslipidemic classification of type 2 diabetic patients. Diabetes Care. 2003;26:2048-51.

28. Sniderman AD. Non-HDL cholesterol versus apolipoprotein B in diabetic dyslipoproteinemia: alternatives and surrogates versus the real thing. Diabetes Care. 2003;26:2207-8.

29. Lu W, Resnick HE, Jablonski KA, Jones KL, Jain AK, Howard WJ, Robbins DC, Howard BV. Non-HDL cholesterol as a predictor of cardiovascular disease in type 2 diabetes: the strong heart study. Diabetes Care. 2003;26:16-23. 
30. Walldius G, Jungner I, Holme I, Aastveit AH, Kolar W, Steiner E. High apolipoprotein B, low apolipoprotein Al, and improvement in the prediction of fatal myocardial infarction (AMORIS study): a prospective study. Lancet. 2001:358:2026-33.

31. Leroux G, Lemieux I, Lamarche B, Cantin B, Dagenais GR, Lupien PJ, Després J-P. Influence of triglyceride concentration on the relationship between lipoprotein cholesterol and apolipoprotein B and Al levels. Metabolism. 2000;49:53-61.

32. von Eckardstein A, Schulte H, Assmann G. Risk for diabetes mellitus in middle-aged Caucasian male participants of the PROCAM study: implications for the definition of impaired fasting glucose by the American Diabetes Association. J Clin Endocrinol Metabol. 2000;85:3101-8.

33. Hwang Y, Ahn H, Kim W, Park C, Park S. Increased apoB/A-I ratio independently associated with type 2 diabetes mellitus: cross-sectional study in a Korean population. Diabet Med. 2012;29:1165-70.

34. Hwang Y-C, Ahn H-Y, Park S-W, Park C-Y. Association of HDL-C and apolipoprotein Al with the risk of type 2 diabetes in subjects with impaired fasting glucose. Eur J Endocrinol. 2014;171:137-42.

35. Assmann G, Gotto AM Jr. HDL cholesterol and protective factors in atherosclerosis. Circulation. 2004;109:|II-8-III-14.

36. Gordon SM, Hofmann S, Askew DS, Davidson WS. High density lipoprotein: it's not just about lipid transport anymore. Trends Endocrinol Metab. 2011; 22:9-15.

37. Kashyap SR, Osme A, Ilchenko S, Golizeh M, Lee K, Wang S, Bena J, Previs SF, Smith JD, Kasumov T. Glycation reduces the stability of ApoAl and increases HDL dysfunction in diet-controlled type 2 diabetes. J Clin Endocrinol Metabol. 2018;103:388-96.

38. Taskinen M-R, BorÚn J. New insights into the pathophysiology of dyslipidemia in type 2 diabetes. Atherosclerosis. 2015;239:483-95.

39. Manjunatha S, Distelmaier K, Dasari S, Carter RE, Kudva YC, Nair KS. Functional and proteomic alterations of plasma high density lipoproteins in type 1 diabetes mellitus. Metabolism. 2016;65:1421-31.

40. Gordon SM, Davidson WS, Urbina EM, Dolan LM, Heink A, Zang H, Lu L, Shah AS. The effects of type 2 diabetes on lipoprotein composition and arterial stiffness in male youth. Diabetes. 2013;62:2958-67.

41. Duvillard L, Pont F, Florentin E, Gambert P, Vergès B. Inefficiency of insulin therapy to correct apolipoprotein Al metabolic abnormalities in non-insulindependent diabetes mellitus. Atherosclerosis. 2000;152:229-37.

42. Watts GF, Barrett PHR, Ji J, Serone AP, Chan DC, Croft KD, Loehrer F, Johnson AG. Differential regulation of lipoprotein kinetics by atorvastatin and fenofibrate in subjects with the metabolic syndrome. Diabetes. 2003;52: 803-11.

43. Pietzsch J, Julius $U$, Nitzsche S, Hanefeld M. In vivo evidence for increased apolipoprotein Al catabolism in subjects with impaired glucose tolerance. Diabetes. 1998;47:1928-34.

44. Wu X, Yu Z, Su W, Isquith DA, Neradilek MB, Lu N, Gu F, Li H, Zhao X-Q. Low levels of ApoA1 improve risk prediction of type 2 diabetes mellitus. J Clin Lipidol. 2017;11:362-8.

45. Walldius $G$, Jungner I. The apoB/apoA-I ratio: a strong, new risk factor for cardiovascular disease and a target for lipid-lowering therapy-a review of the evidence. J Intern Med. 2006;259:493-519.

46. Schianca GC, Pedrazzoli R, Onolfo S, Colli E, Cornetti E, Bergamasco L, Fra G, Bartoli E. ApoB/apoA-I ratio is better than LDL-C in detecting cardiovascular risk. Nutr Metab Cardiovasc Dis. 2011;21:406-11.

47. Mao Y, XU Y, Lu L. The nonlinear association between apolipoprotein B to apolipoprotein A1 ratio and type 2 diabetes. Medicine. 2017;96:1-4.

48. Janghorbani M, Amini M. Utility of serum lipid ratios for predicting incident type 2 diabetes: the Isfahan diabetes prevention study. Diabetes Metab Res Rev. 2016;32:572-80.

49. Thompson A, Danesh J. Associations between apolipoprotein B, apolipoprotein $\mathrm{Al}$, the apolipoprotein $\mathrm{B} / \mathrm{Al}$ ratio and coronary heart disease: a literature-based meta-analysis of prospective studies. J Intern Med. 2006; 259:481-92.

50. Hwang $\mathrm{Y}-\mathrm{C}$, Ahn H-Y, Park S-W, Park C-Y. Apolipoprotein B and non-HDL cholesterol are more powerful predictors for incident type 2 diabetes than fasting glucose or glycated hemoglobin in subjects with normal glucose tolerance: a 3.3-year retrospective longitudinal study. Acta Diabetol. 2014;51: 941-6.

51. Hirst K. TODAY study group lipid and inflammatory cardiovascular risk worsens over 3 years in youth with type 2 diabetes: the TODAY clinical trial. Diabetes Care. 2013;36:1758-64.
52. Jing F, Mao Y, Guo J, Zhang Z, Li Y, Ye Z, Ding Y, Wang J, Jin M, Chen K. The value of Apolipoprotein B/Apolipoprotein $\mathrm{A} 1$ ratio for metabolic syndrome diagnosis in a Chinese population: a cross-sectional study. Lipids Health Dis. 2014;13:81.

53. Jun JE, Choi YJ, Lee Y-H, Kim DJ, Park SW, Huh BW, Lee EJ, Jee S-H, Hur KY, Choi $\mathrm{SH}$. ApoB/ApoA-I ratio is independently associated with carotid atherosclerosis in type 2 diabetes mellitus with well-controlled LDL cholesterol levels. Korean J Intern Med. 2018;33:138.

54. Park KH, Shin DG, Kim JR, Hong JH, Cho KH. The functional and compositional properties of lipoproteins are altered in patients with metabolic syndrome with increased cholesteryl ester transfer protein activity. Int J Mol Med. 2010;25(1):129-36.

55. Holvoet $P$, Harris TB, Tracy RP, Verhamme P, Newman AB, Rubin SM, Simonsick EM, Colbert LH, Kritchevsky SB. Association of high coronary heart disease risk status with circulating oxidized LDL in the well-functioning elderly: findings from the health, aging, and body composition study. Arterioscler Thromb Vasc Biol. 2003;23:1444-8.

56. Itabe H. Oxidative modification of LDL: its pathological role in atherosclerosis. Clin Rev Allergy Immunol. 2009;37:4-11.

57. Njajou OT, Kanaya AM, Holvoet P, Connelly S, Strotmeyer ES, Harris TB, Cummings SR, Hsueh WC. Association between oxidized LDL, obesity and type 2 diabetes in a population-based cohort, the health, aging and body composition study. Diabetes Metab Res Rev. 2009;25:733-9.

58. Wang JC, Bennett M. Aging and atherosclerosis: mechanisms, functional consequences, and potential therapeutics for cellular senescence. Circ Res. 2012:111:245-59.

59. Ganjifrockwala F, Joseph J, George G. Serum oxidized LDL levels in type 2 diabetic patients with retinopathy in Mthatha region of the eastern Cape Province of South Africa. Oxidative Med Cell Longev. 2016;2016:1-8.

60. Monickaraj F, Aravind S, Gokulakrishnan K, Sathishkumar C, Prabu P, Prabu D, Mohan V, Balasubramanyam M. Accelerated aging as evidenced by increased telomere shortening and mitochondrial DNA depletion in patients with type 2 diabetes. Mol Cell Biochem. 2012;365:343-50.

61. Motamed M, Nargesi AA, Heidari B, Mirmiranpour H, Esteghamati A, Nakhjavani M. Oxidized low-density lipoprotein (ox-LDL) to LDL ratio (ox$\mathrm{LDL} / \mathrm{LDL}$ ) and ox-LDL to high-density lipoprotein ratio (ox-LDL/HDL). Clin Lab. 2016;62:1609-17.

62. Viigimaa M, Abina J, Zemtsovskaya G, Tikhaze A, Konovalova G, Kumskova E, Lankin V. Malondialdehyde-modified low-density lipoproteins as biomarker for atherosclerosis. Blood Press. 2010;19:164-8.

63. Mooradian AD. Dyslipidemia in type 2 diabetes mellitus. Nat Rev Endocrinol. 2009:5:150.

\section{Publisher's Note}

Springer Nature remains neutral with regard to jurisdictional claims in published maps and institutional affiliations.
Ready to submit your research? Choose BMC and benefit from:

- fast, convenient online submission

- thorough peer review by experienced researchers in your field

- rapid publication on acceptance

- support for research data, including large and complex data types

- gold Open Access which fosters wider collaboration and increased citations

- maximum visibility for your research: over $100 \mathrm{M}$ website views per year

At BMC, research is always in progress.

Learn more biomedcentral.com/submissions 